\title{
Theoretical study of the electric and the magnetic dipole in UV-Vis and ECD of fluralaner: the folded conformation and the extended conformation
}

kamal ziadi ( $\boldsymbol{\sim}$ kamel.ziadi@univ-batna.dz )

Batna1 university, Department of Chemistry https://orcid.org/0000-0001-5254-5879

\section{Research Article}

Keywords: Electronic Transition, ECD, transition density matrix, charge transfer, Fluralaner

Posted Date: March 11th, 2021

DOl: https://doi.org/10.21203/rs.3.rs-261959/v1

License: (c) (1) This work is licensed under a Creative Commons Attribution 4.0 International License.

Read Full License 


\title{
Theoretical study of the electric and the magnetic dipole in UV-Vis and ECD of fluralaner: the folded conformation and the extended conformation
}

\author{
Kamal.Ziadi \\ Department of chemistry \\ LCMVAR laboratory, Faculty of sciences of matter, Batna 1 University, Batna, Algeria
}

The corresponding author: Kamal Ziadi

The e-mail address: kamel.ziadi@univ-batna.dz

Tel:213 675051466

\begin{abstract}
:
Through our contribution, we have carried out the theoretical study of the transition characteristics of one-photon absorption (OPA) spectra of the folded conformation and the extended conformation of fluralaner. The electronic transitions in OPA are visualized with charge difference density (CDD) and transition density matrix (TDM) to explain the charge transfer via hole-electron distribution. We've also analyzed the transition dipole electric/ magnetic moment by using the iso surface (real space) and TDM diagram in order to determine the portions of molecules, which have the most contribution in ECD spectra.
\end{abstract}

Key words: Electronic Transition, ECD, transition density matrix, charge transfer, Fluralaner.

\section{Introduction}

Fluralaner [1-3], 4-[(5R)-5-(3, 5-dichlorophenyl)-5-(trifluoromethyl)-4H-1, 2-oxazol-3-yl] -2-methyl$\mathrm{N}$ - [2-oxo-2-(2, 2, 2-trifluoroethylamino) ethyl] benzamide, is a systemic acaricidal and insecticide oral administration. It is also used to repel ticks for dogs and fleas for cats. It is a broad-spectrum veterinary medicine affiliating to isooxazoline insecticides that acts by interacting with the $\gamma$ aminobutyric acid receptor (GABAR) in pest control. The US Food and Drug Administration has certified it under the trade name Bravecto for dog's treatment for fleas on May 2014, and the EU did the same on February 2014. Our study focus on tow conformer of fluralaner; the folded conformation and the extended one (figure 1).

In quantum chemistry, the description of the electronic structure induced by light, from the fundamental state to the excited one, is habitually involved in researching electronic transitions of molecule. In fact, it has previously been shown that the accurate and the best methodology for describeing the light-induced electronic structure reorganization is the most important for the improvement of new theoretical methods or new experimental techniques. In this field, a considerable attention has been drawn in the literature [4-11]. The Time Dependent Density Functional Theory 
(TDDFT) is a really successful theory of electronic structure of matter, where the TDDFT has drawn a considerable attention in the literature [7, 11-24] for calculating the transitions energy with relative intensity. According to this computation, many of objects are resulting such as: the difference in density matrix, which is known as the difference between the excited and ground states density matrix expressed on the basis set of atomic functions. In the transition process, charge density difference (CDD) analysis is prevalently used and commonly accepted method for studying the difference in charge distribution between two-electron states of a system. Our work admits a variety of analysis methods, according to the density difference between the excited state and the ground one. for unveiling the basic nature of an electron excitation, The transition density matrix (TDM) is very useful. TDM falls into two forms: i) the three-dimensional real space form, which can be expressed through drawing an isosurface map. A large value at a position corresponds to a large overlap of hole and electron, this reflect the contribution at this place taken into consideration. ii) The matrix form, in 2D, this TDM form can be exhibited as heat map, namely color-filled matrix map, which may be atom-based (our work) or fragment-based. This approach (TDM) is also considered as the best performing method for determining all of the one-electron properties such as: the transition dipole moment and the atomic transition charge [25-31].

Through TDM map, much information can be obtained, and different transition types can be distinguished (local excitation and charge transfer excitation) [27, 30, 32]. In this paper, we've begun with defining the theoretical background of the electronic transition in UV-Vis and ECD spectra. After that, we've studied charge transfer, by using method of charge density difference (CDD) to illustrate the distinction in electron density during excitement state (electron-hole coherence) through i) the transition density matrix using 2D visualization, where an increase in electron density is described at the level of the electrons, and a decrease in electron density is described at the level of the holes ii) the charge difference densities using 3D visualization (iso surface). Finally, we've visualized the transition electric dipole moment density and the transition magnetic dipole moment density of the excited states that have the strongest rotator strength using TDM in 2D and in real space 3D.

\section{Brief theory}

\section{Oscillator strength $f$ :}

For occurring, an oscillating dipole must be induced by interaction of the molecules electric field with the electromagnetic radiation. For an electronic transition in theoretical chemistry filed, the oscillator strength $f$ is always used for representing transition strength in UV-Vis spectrum, and is defined as:

$$
f=\frac{2}{3} \Delta \varepsilon_{i}\left(D_{i x}^{2}+D_{i y}^{2}+D_{i z}^{2}\right)
$$


where $\Delta \varepsilon_{i}$ denotes the transition energy between the two electronic states. The three Cartesian components of transition electric dipole moment between ground state and an excited state can be calculated as follows [27-31]:

$$
D_{x}^{\mu}=P_{\mu \mu}^{\operatorname{tran}}\left\langle\chi_{\mu}|-x| \chi_{\mu}\right\rangle+\frac{1}{2} \sum_{\mu \neq v}\left[P_{\mu v}^{\operatorname{tran}}\left\langle\chi_{\mu}|-x| \chi_{v}\right\rangle+P_{v \mu}^{\operatorname{tran}}\left\langle\chi_{v}|-x| \chi_{\mu}\right\rangle\right]
$$

Where $P_{v \mu}^{\text {tran }}$ is the transition density matrix in basis set.

\section{Transition density matrix TDM}

To evaluate the electronic excitation, the TDM between ground and an excited state can be calculated as [27-31]

$$
P_{v \mu}^{\text {tran }}=\sum_{i}^{o c c} \sum_{j}^{v i r} w_{i}^{j} C_{\mu i} C_{v j}
$$

$w_{i}^{j}$ corresponds to coefficient of the configurations involved in the excitation, $C_{\mu i}$ denotes the expansion coefficient of basis set function $\mu$ in orbital molecule $i$. This permits to calculate the atomic contribution of the transition electric dipole moment. It should be noted that the TDM is in real representation, which can be constructed via TDM in basis function representations

$$
T\left(r ; r^{\prime}\right)=\sum_{\mu} \sum_{v} P_{\mu v}^{\text {tran }} \chi_{\mu}(r) \chi_{v}\left(r^{\prime}\right)
$$

The excitation can be absolutely represented as $\mathrm{I} \rightarrow \mathrm{j}$ molecule orbital transition and suppose that there are only two basis set functions, the TDM could explicitly be written as below form:

$$
P_{\mu v}^{\text {tran }}=P_{A B}^{\text {tran }}=\left[\begin{array}{ll}
C_{A i} C_{B j} & C_{A i} C_{B j} \\
C_{A i} C_{A j} & C_{B i} C_{A j}
\end{array}\right]
$$

Where $\mu$ and $v$ denote the basic functions centered at atom $A$ and on $B$.

If off-diagonal element $P_{\mu v}^{\text {tran }}$ has a big magnitude, we can say that, during the excitation, the atom $A$ and the atom $B$ have large contribution to hole and electron, respectively. The transition density is contributed by different basic functions (different atomic), that can be considered as the transition visualized by this transition density as a charge transfer excitation. If on-diagonal element $P_{\mu, \mu}^{\operatorname{tran}}$ has a large magnitude, it implies that basis function $\mu$ must have large contribution to both hole and electron. This reflects the local excitation characteristics.

\section{Electronic circular Dichroism ECD}

Theoretically [33], the rotatory strength of an ECD (ECD spectroscopy characterizes the chiral molecules) is the imaginary part of the scalar of the scalar product of the transition electric dipole moment $D_{0 n}$ and the transition magnetic dipole moment $M_{0 n}$

$$
R_{0 n}=\operatorname{Im}\left[D_{0 n} \cdot M_{0 n}\right]
$$

The transition electric dipole moment $D_{0 n}=D_{n 0}=D_{0 n}^{*}$, whereas the transition magnetic dipole moment $M_{0 n}=-M_{0 n}^{*}$

The transition electric dipole moments of different Cartesian components is defined as follows 


$$
M_{x}^{\mu}=P_{\mu \mu}^{\operatorname{tran}}\left\langle\chi_{\mu}\left|L_{x}\right| \chi_{\mu}\right\rangle+\frac{1}{2} \sum_{\mu \neq v}\left[P_{\mu v}^{\operatorname{tran}}\left\langle\chi_{\mu}\left|L_{x}\right| \chi_{v}\right\rangle+P_{v \mu}^{\operatorname{tran}}\left\langle\chi_{v}\left|L_{x}\right| \chi_{\mu}\right\rangle\right]
$$

Knowing that the operator for magnetic dipole moment, due to movement of electrons, is the angular momentum operator L, where $L_{x}=y \frac{\partial}{\partial z}-z \frac{\partial}{\partial y}$.

\section{Computational details}

The ab initio calculations of UV-Vis and ECD of folded conformer extended conformer of fluralaner molecules using the time-dependent density function theory (TDDFT) methodology have first been reported. The geometry optimizations have been carried out using Gaussian9 program [34] at the B3LYP/6-31G* level to make sure of the minimum energy of molecular geometry. The excitation analysis has been predicted using the CAM-B3LYP functional and 6-31G* basis sets [35]. The CAM-B3LYP [36] has been utilized, in our work, since it is suitable to study charge transfer excitations. The transition density matrix (TMD), the charge difference densities (CDD) presented by heat maps (electron hole), and the transition electric dipole/ magnetic moment have been carried out by the Multiwfn 3.8 program [37]. Density in real space (3D iso surface) is designed by means of VMD software [38] to obtain preferable visualization effect.

\section{Resultants and discussion}

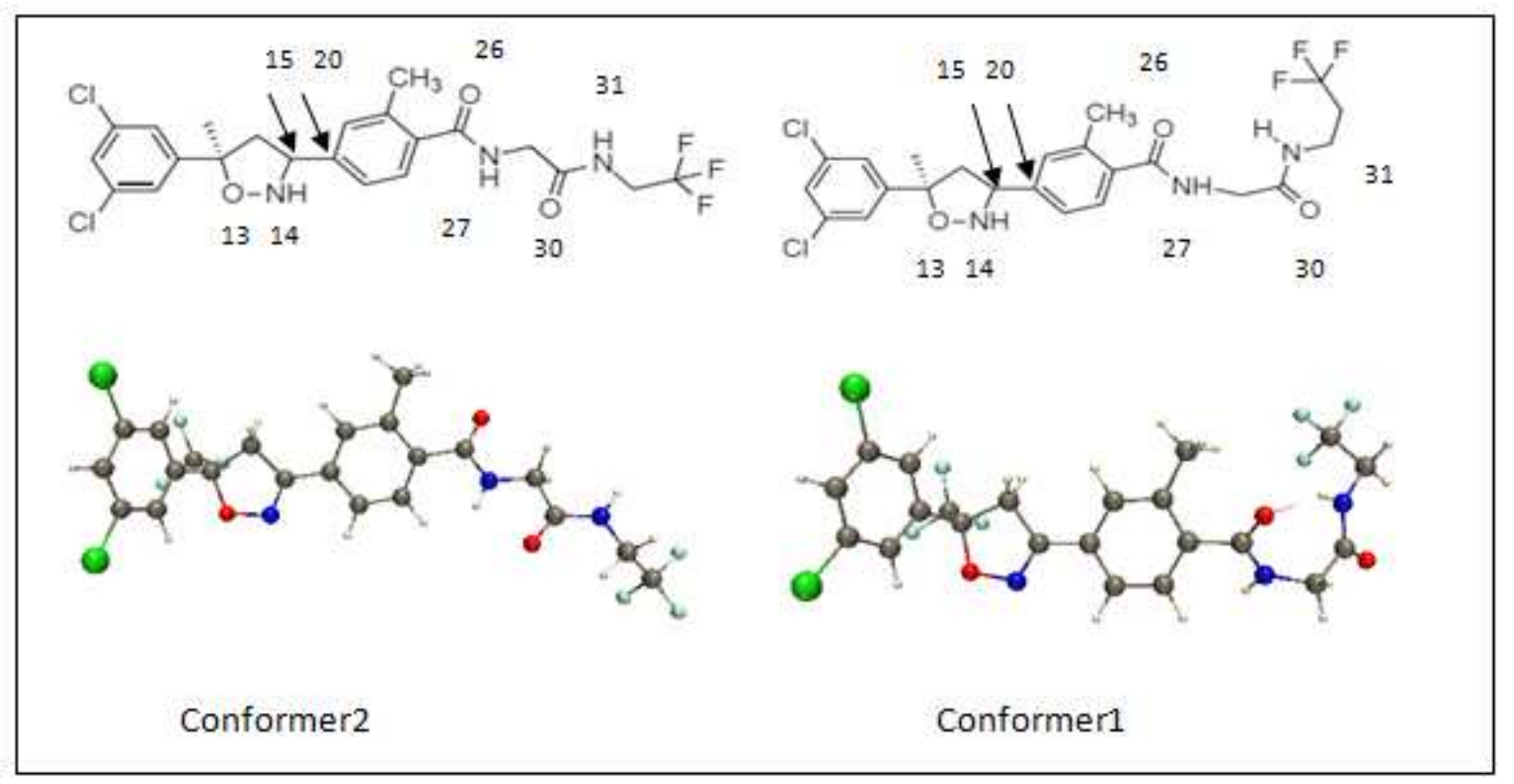

Figure.1. Molecular structure of 4-[(5R)-5-(3, 5-dichlorophenyl)-5-(trifluoromethyl)-4H-1, 2-oxazol3-yl] -2-methyl-N- [2-oxo-2-(2, 2, 2-trifluoroethylamino) ethyl] benzamide.

\section{Electronic Transition and ECD Spectrum}

Figure 3 is shown the calculated UV-Vis spectra whereas our work focused on the strong intensity of the one photon transition that presented in our work by oscillator strength. From the feature in the figure 2, the absorption spectrum (UV-Vis) of the two conformers is mainly divided of two parts. The first part is strongest oscillator strength peaks are founded in lowest energy between 300-260 nm, which 
corresponds to the excitation states S1, S2 and S5 for conformer1 and S1, S2 and S3 for conformer2. Compared with the first part of excited state, the oscillator strength of the second part of excited state is considerably weaker.
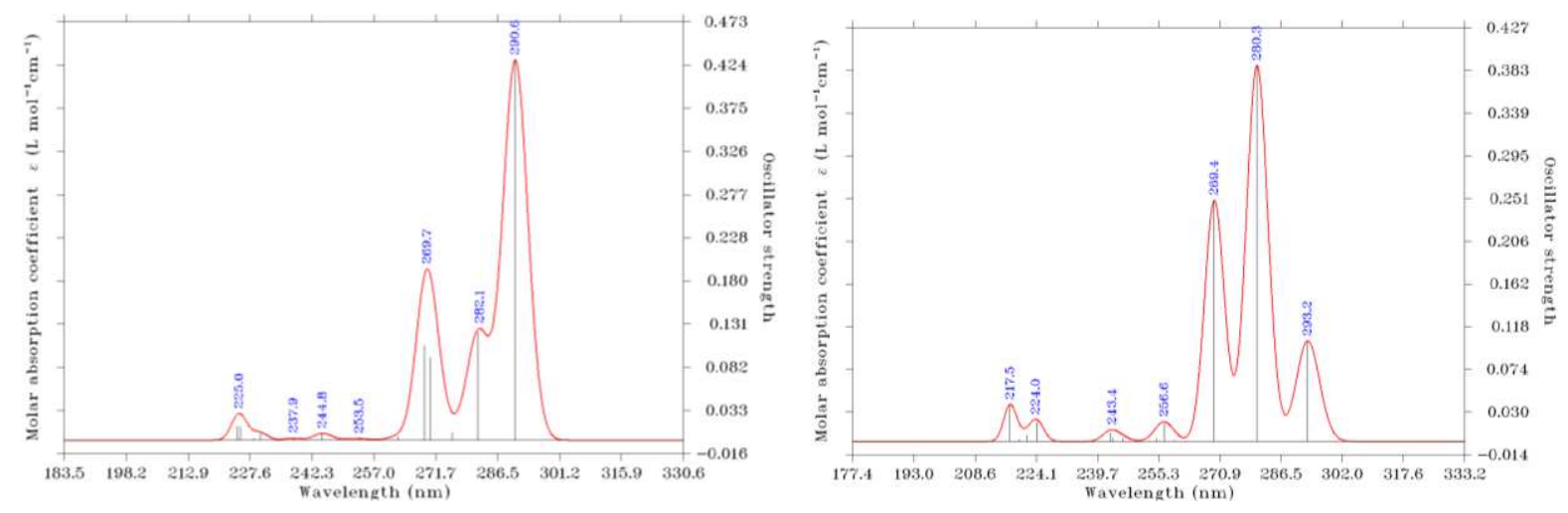

Figure.2. calculated UV-Vis spectra of conformer1 (left) and conformer2 (right)
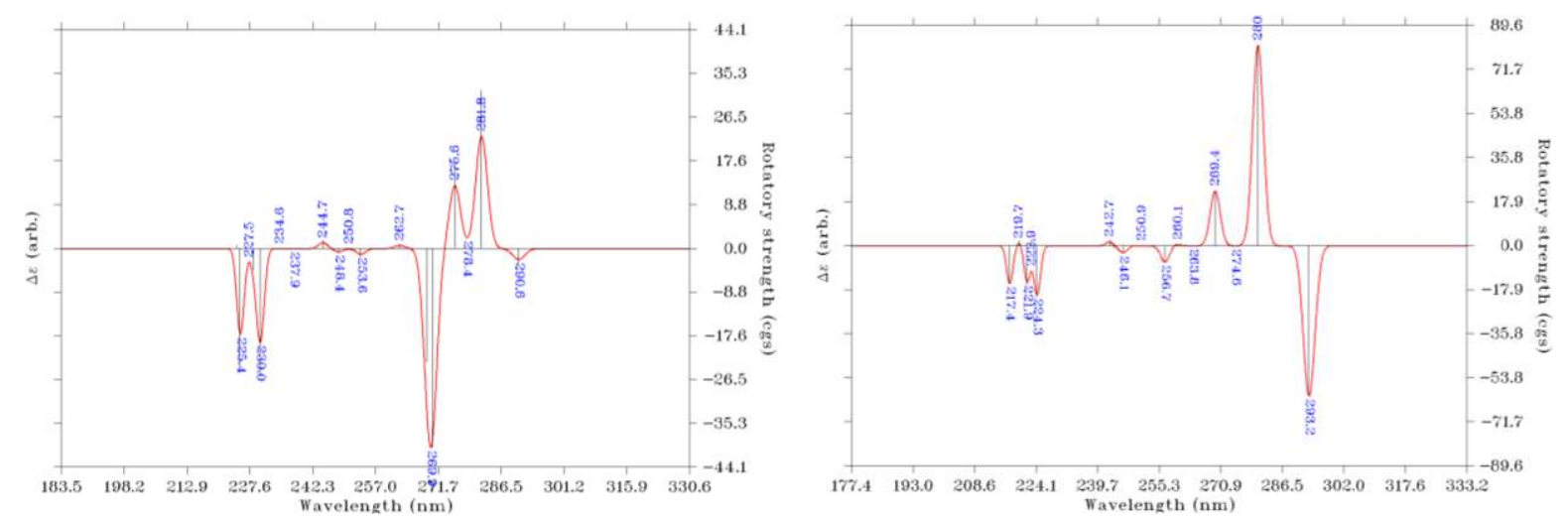

Figure.3. calculated ECD spectra of conformer1 (left) and conformer2 (right)

Concerning ECD spectra, we can observe that ECD intensity of conformer2 is stronger than conformer1. According to the ECD spectrum of conformer1 in figure 3, the S2 and S4 are characterized by big peaks, strong rotator strength, and opposite directions. According to the ECD spectra of conformer2 in figure 3, the excited states S1 and S2 have the strongest intensity in the rotator strength peaks of the absorption spectrum, hence the four excited states S1, S2 (two peaks) and S4 are chosen in our study. According to Eq. (6), it can be observed that the difference of the absorption spectrum in the ECD spectra is due to the different electric-magnetic interactions within the molecule, when it is excited by light. According to Eq. (6), the calculations of the moment tensor product are shown in table 1. According to the results of tensor product, we've checked that S2 is positive and $\mathrm{S} 4$ is negative. concerning the conformer2, the calculations have also shown the sing of both $\mathrm{S} 1$ and $\mathrm{S} 2$, where we can observe that $\mathrm{S} 1$ is negative and $\mathrm{S} 2$ is positive, which is consistent with the ECD spectrum in figure 3. 


\section{Charge difference density (CDD) via electron-hole}

According to the results of UV-Vis spectra in section above, it should be very interesting to study charge transfer via electron hole analysis in S1, S2, and S5 exited state of conformer1 and S1, S2, and $\mathrm{S} 3$ of conformer2 in one photon transition. For this purpose, we have plotted the difference map of transition density matrix and charge difference densities that represent the electron-hole distribution diagram of conformer1 and 2. Electron excitation can be considered as hole-electron transition. we must first study $\mathrm{X}$-axis (hole position), and then $\mathrm{Y}$-axis (electron position). According to the colors in the heat map (TDM), the all of off-diagonal elements have large magnitude as shown in figure 4 and figure 5, it indicates that the transition represented this transition density can be considered as charge transfer excitation. These findings are consistent with the situation exhibited by the isosurface.

Table 1. The tensor product of transition electric and magnetic for conformer1 and conformer2 in ECD

\begin{tabular}{|l|cc|r|r|r|}
\hline molecule & \multicolumn{2}{|l|}{ Excited state } & \multicolumn{1}{c|}{$\boldsymbol{D}$} & \multicolumn{1}{c|}{$\boldsymbol{M}$} & $\boldsymbol{D} \times \boldsymbol{M}$ \\
\hline & & $\mathrm{X}$ & 1.0298 & 0.0370 & \\
& $\mathrm{~S} 2$ & $\mathrm{Y}$ & -0.0285 & -0.3104 & 0.1285 \\
& & $\mathrm{Z}$ & 0.3429 & -0.5117 & \\
& & $\mathrm{X}$ & 0.6381 & 0.2108 & \\
& $\mathrm{~S} 4$ & $\mathrm{Y}$ & -0.1194 & -0.2777 & -0.1182 \\
& & $\mathrm{Z}$ & 0.1459 & -0.3388 & \\
& & $\mathrm{X}$ & -0.8326 & -0.0923 & \\
& $\mathrm{~S} 1$ & $\mathrm{Y}$ & -0.2311 & -0.4384 & -0.3032 \\
& & $\mathrm{Z}$ & 0.2140 & 0.5844 & \\
Conformer & & & & \\
& & $\mathrm{X}$ & 1.4891 & -0.2198 & \\
& $\mathrm{~S} 2$ & $\mathrm{Y}$ & 0.2965 & 0.3692 & 0.1894 \\
& & $\mathrm{Z}$ & -0.0328 & -0.8648 & \\
\hline
\end{tabular}

The magnitude of diagonal element in the all TDM is quite little, but in some cases, the diagonal terms has a large magnitude, which means that the present electron excitation does not principally occur in the electron transfer between different atoms or groups. The main feature of this excitation is the local excitation like: the regions that are near to $(26,26)$ are shown in a very red color in almost excitation.

According to the first preliminary analysis of electron-hole distribution and transition density matrix (TDM) in Fig. 4 and 5 of one-photon excited states, we conclude that all excitations occur in the half of the molecules i.e. between O13-O30 ( Conformers 1) and O13-O26 ( Conformers 1)

Through studying the transition density matrix of electronic transition, we will be able to know how electrons transfer is among different sites. In figure 4-S1, concerning the first excitation S1 of conformer1, we can observe the hole position (X-axis) is mostly strong in O-30 atom. It can be observed, from the Y-axis of the heat map in figure 4-S1, that the electron position is mainly 
concentrated on the N-14, C-15, C-18 atoms, and a lesser extent on C-25 atom. So, we can confirm that the charge is transferred from $\mathrm{O}-30$ atom to $\mathrm{N}-14, \mathrm{C}-15, \mathrm{C}-18$ and $\mathrm{C}-25$ atoms. The same remark, concerning S2 in figure 4, but the hole positions become two sites O-30 and O-26. The transition density matrix of S5, the electron transfer to the middle portion of the molecule, like as S1 and S2, but the electrons come from dichloropheny group. it can be confirmed, through the transition density matrix of Figure 4-S5, that the hole density is concentrated on the dichloropheny group in the right of the molecule.
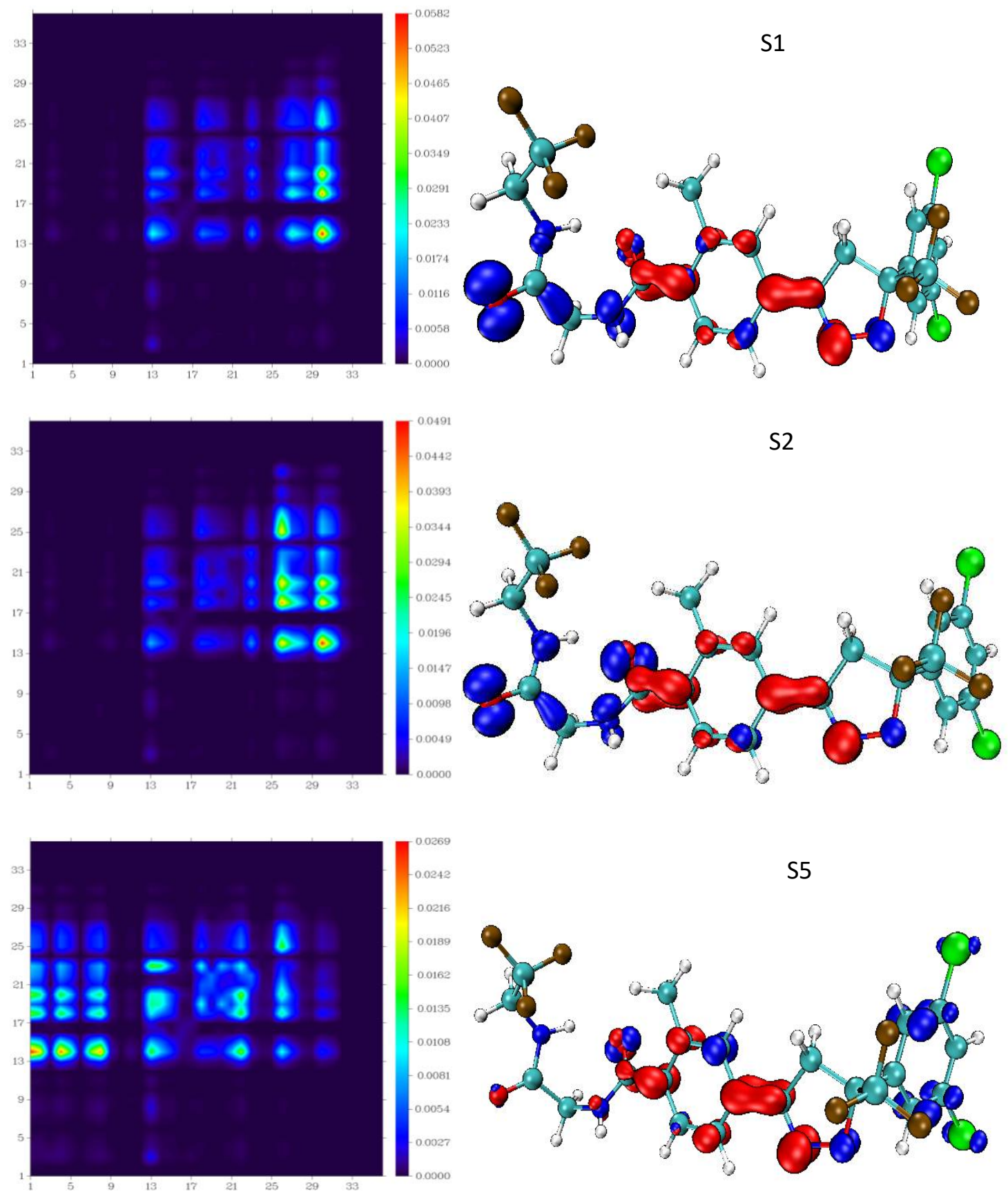

Figure.4. Transition density matrix (TDM) and charge difference density (CDD) of excited states S1, S2, S5 of conformer1. The isosurface color of the electron density is red while the bleu is hole density. 
The TDM and CDD, in figure 4, represent electron transition information between ground and excited states via electron-hole distribution. According to figure 4, we observe that the electron is distributed on the N-14 atom, band between oxazol and benzamide exactly C15-C20 and band C18-C25 in benzamide, and the hole is distributed on the $\mathrm{O}-13, \mathrm{O}-16, \mathrm{~N} 27$ and greater on $\mathrm{O}-30$, which indicates that the electron is transferred from the all oxygen atoms and the $\mathrm{N}-27$ to the $\mathrm{N}-14$ atom , the band C15-C20 and the band C18-C25 in benzamide. CDD of S5 is similar to those of S1 and S2, except for the hole distribution, whereas we can see the electron is transferred from the right of molecule (dichloropheny group) instead of O-30 and N-27 to half molecule (N-14 atom, the band C15-C20 and the band $\mathrm{C} 18-\mathrm{C} 25$ in benzamide).
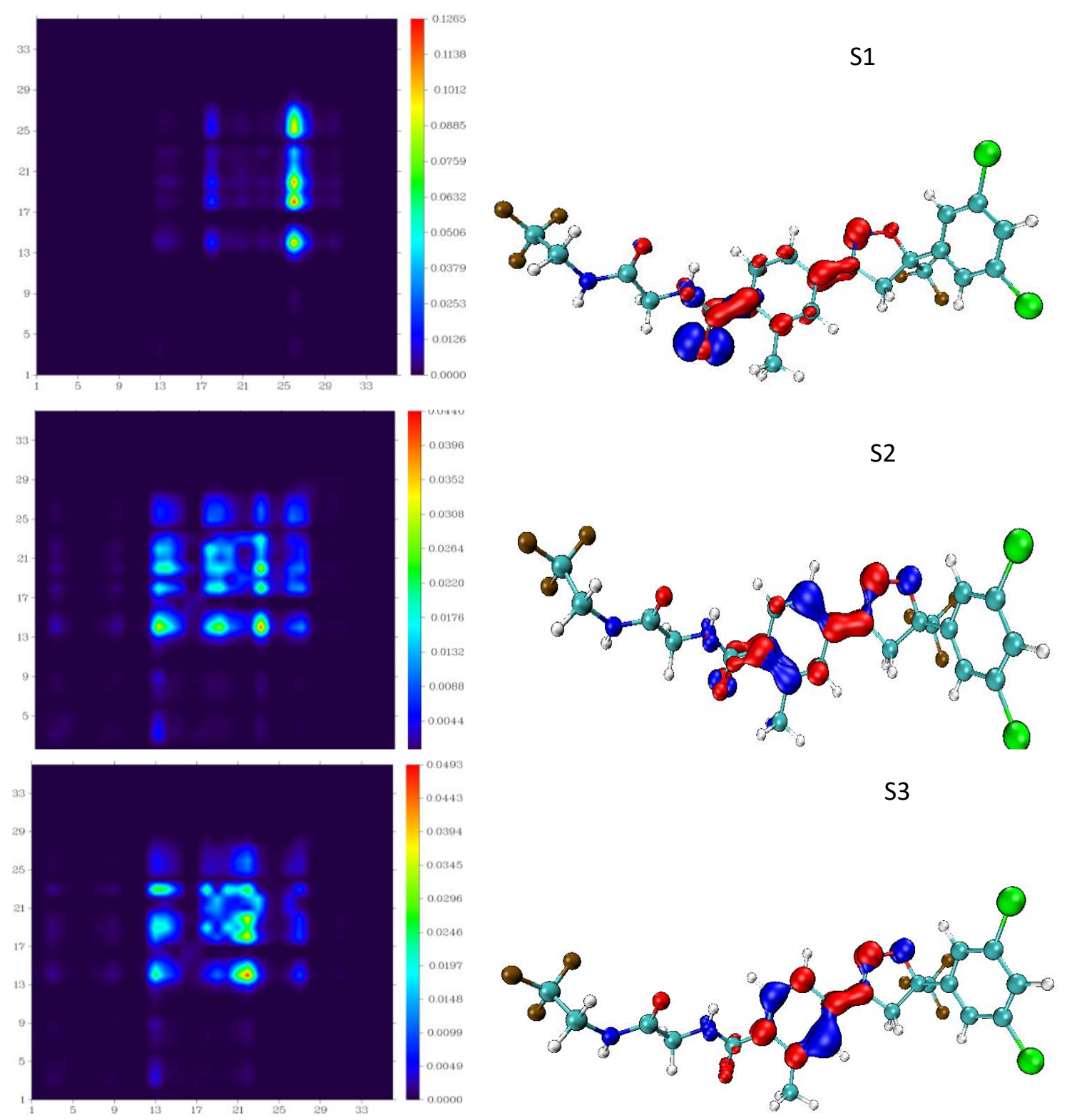

Figure.5. Transition density matrix (TDM) and charge difference density (CDD) of excited states S1, $\mathrm{S} 2, \mathrm{~S} 3$ of conformer2. The isosurface color of the electron density is red while the bleu is hole density. 
Next, we will analyze the first three excited states of conformer2 for recognizing the differences in electronic transitions between the extended conformer and the folded conformer. At the first excitation $\mathrm{S} 1$, as shown in the figure 5 TDM map, it is noted that there is a strong electron transfer from the O-26 atom on benzamide group to the $\mathrm{N}-14, \mathrm{C}-15$ atoms (oxazol group) and the region of $\mathrm{C}-18 \sim \mathrm{C}-25$ (benzamide group), this remark totally agrees with the hole-electron isosurface map, namely there is a large bleu isosurface (hole density) at O-26 atom and red isosurface (electron density) in the middle molecules especially the region of atoms $\mathrm{N}-14 \sim \mathrm{C}-15$ and C-18 C-25. In addition, according to the iso surface map (figure 5), we can observe that O-30 atom is not surrounded by any isosurface, which means that this atom does not transfer electrons in contrast to conformer1; this observation is the most different between conform 1 and conformer2. The second and third excited state in the one -photon transition of the conformer2 is S2 and S3 as shown in figure 5. According to TDM and electron-hole iso surface of second transition $\mathrm{S} 2$, it has been found that the O-13, C-23 and C-19 can provide electrons, so those electrons transformed to O-13, C-23, and C-19. The third transition S3 is similar to $\mathrm{S} 2$, but it can be observed that the distribution of electrons and holes is opposite on benzamide ring, whereas in S3, in contrast to S2, the electrons transfer from C-23 and C-19 to C-23 and C-19 in the ring. Finally, we can observe that the hole and electron occupy similar spatial region (local excitation).

\section{Electric and magnetic in ECD spectrum}

To study the contribution of the transition electric/magnetic dipole moment, we've carried out the visualization of their densities (dipole/ magnetic) for the (S2, S4) for conformer1 and the (S1, S2) and conformer2 in ECD. The figures 6, 7, 8, and 9 have been shown the transition electric/ magnetic dipole moment densities of the excites states S4, S2 (two excited) and S1 respectively, where red and blue iso surface present positive and negative parts in transition electric dipole moment densities, while the green and mauve iso surface present positive and negative parts in transition magnetic dipole moment densities. There are many points of the figures appear very blue color or very red showing that corresponding atoms and neighboring ones have significant negative or positive contributions, respectively. These areas are also evidently reflected in the iso surface map of transition electric dipole/magnetic moment density. According to the figure-6, we may say that the contribution of $D_{x}$ is bigger than both of $D_{y}$ and $D_{z}$ to the transition electric dipole moment.

The component- $X$ of the transition electric dipole moment reveals that the electron-hole density is approximately distributed everywhere in the molecule, while the component- $\mathrm{X}$ and $-\mathrm{Y}$ of electronhole density is concentrated in middle molecule. It can be also remarked, in each component in figure 6 , that the strength of transition magnetic dipole moment is significantly enhanced. The transition magnetic dipole moments density of the X-, Y-, and Z-component corresponding to the S2 excited state are concentrated on oxazol group, benzamied group, and both of O-30 and N-31 atoms. The figure 7 shows that the contribution of both $D_{x}$ and $D_{y}$ is larger than $D_{z}$, while $M_{z}$ and $M_{y}$ contribute 
the mostly to the transition magnetic dipole moment. The ichlorophenyl group , oxazol, especially (O13, N-14), benzamide group and O-30 atom contribute nearly to all the transition electric dipole moments density of the X-component and the transition magnetic dipole moments density of the $\mathrm{Y}$-, Z-component.
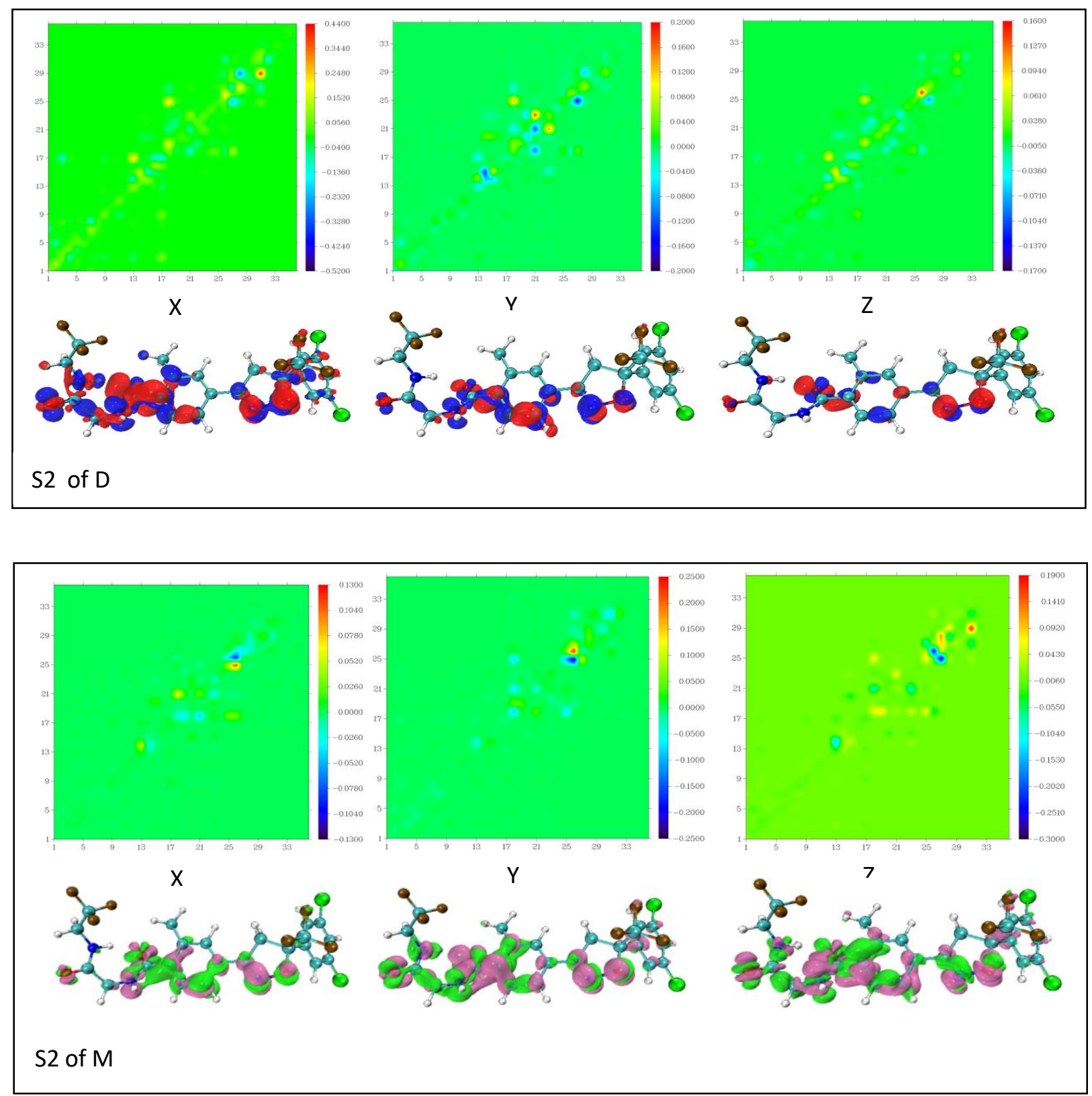

Figure.6. Transition electric dipole moment density (above) and transition magnetic dipole moment density (below) for excited state S2 in ECD of conformer1.

The transition electric/ magnetic dipole moment density map in 2D and 3D of S1 (conforme2) are displayed in figure 8. From the heat maps (TDM), it's clear to know that the contribution of both $D_{x}$ and $D_{y}$ is larger than $D_{z}$, while the contribution of both $M_{y}$ and $M_{z}$ is larger than $M_{x}$. The density matrix map and isosurface of transition dipole moment density show that the contribution of electric 
transition of X component comes principally from O-13, N-14 atoms on oxazol, and N27-C25-O26 on benzamide.
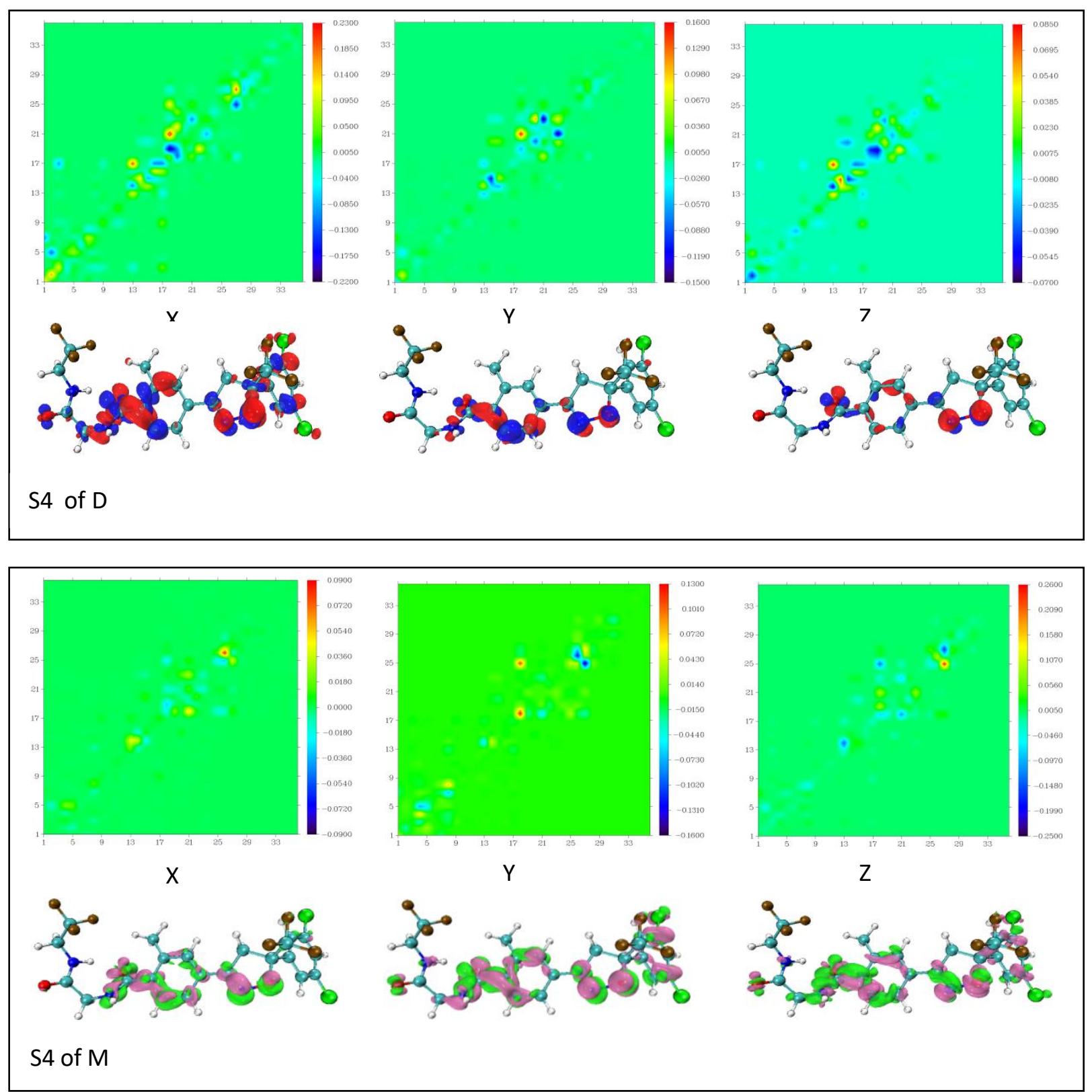

Figure.7. Transition electric dipole moment density (above) and transition magnetic dipole moment density (below) for excited state S4 in ECD of conformer1.

Concerning the transition magnetic dipole moment of Y-component, the contribution comes from N27-C25-O26 on benzamide. From the transitions of X-component and Z-component, we can observe that some sites in the parts of oxazol, and N27-C25-O26 on benzamide are obviously strong, which means that coupling between the atoms has a significant contribution to the transition dipole moment of X- and Z-component. We remark that the transition magnetic dipole moment of N27-C25-O26 to Y-component shows mainly a negative contribution (the regions near $(27,27)$ and $(26,26)$ show a 
very blue color), while $\mathrm{N} 27-\mathrm{C} 25-\mathrm{O} 26$ reveal a positive contribution (the regions near $(27,27)$ and $(26$, 26) show a very red color).
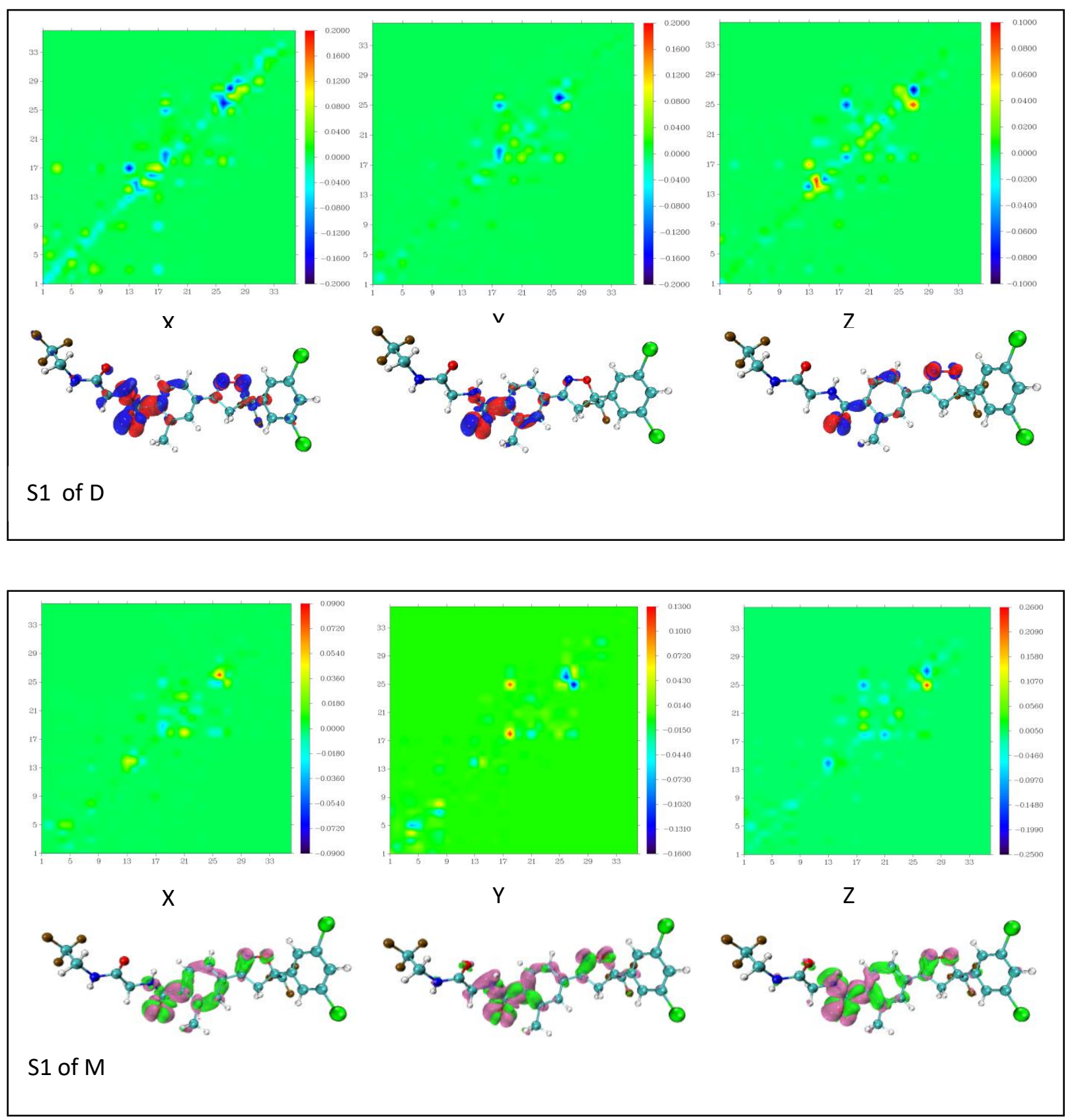

Figure. 8. Transition electric dipole moment density (above) and transition magnetic dipole moment density (below) for excited state S1 in ECD of conformer2.

According to the figure 9, it can be remarked that the contribution of transition electric dipole moment is mainly contributed by $D_{x}$ with an acceptable contribution of both $D_{y}$ and $D_{z}$. We can observe that the most areas of (X-and Y-component) of the heat map are red, so the red matrix element contributes more positively to the transition dipole moment of X-and Y-component. The transition electric dipole 
moment of the $\mathrm{X}$-component is almost distributed in the all molecule and has the biggest intensity, except for dichlorophenyl group and trifluoromethyl group, that show only weak interactions.
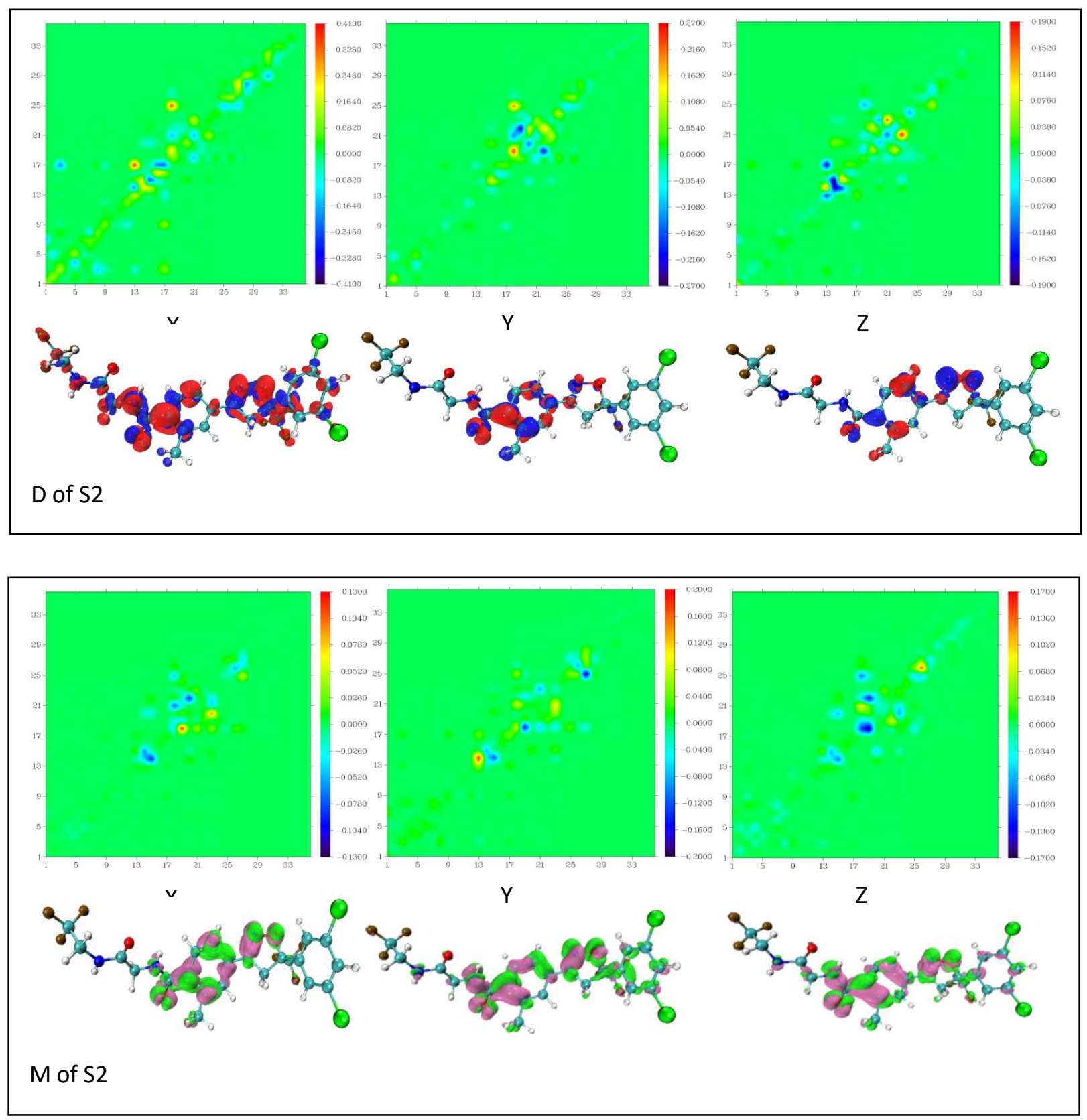

Figure.9. Transition electric dipole moment density (above) and transition magnetic dipole moment density (below) for excited state S2 in ECD of conformer2.

According to transition magnetic dipole moment density, it can be found that the strength of $\mathrm{X}, \mathrm{Y}$ and $\mathrm{Z}$ component is very similar (the $\mathrm{X}$ component is only a little smaller), and the positive and negative isosurface strength of S1 excited state distribution in oxazol and benzamide location is bigger than that of $\mathrm{S} 2$. 


\section{Conclusion}

The electronic transitions of conformer1 and conformer2 are theoretically investigated using optical spectra of one photon absorption and electronic circular dichroism. The 2D (TDM) and 3D (iso surface) representations for photon absorption on electronic transitions between ground and excited states have theoretically been studied. The transition dipole moment, the charge distribution, and the electron-hole consistency of molecules (the folded structure and the extended structure of fluralaner) on electronic transitions have been visualized. According to the confomer1 visualizations, we can say that the electron is transferred from the $\mathrm{O}-30$ and $\mathrm{O}-13$ to $\mathrm{N}-14$ atom and benzamide and oxazol group, while the conformer2, except the first excitation S1, (the hole is distributed on the O-26), and according to the graph of figure5, we can immediately recognize that this is a local excitation, since most of the part of both hole and electron are distributed on the portion consisted of benzamide and oxazol group. In ECD spectrum, the intensity of peaks can be determined by tensor product calculation of transition dipole/ magnetic moment. The contributions to the transition electric and magnetic dipole moment densities are mostly focused on the benzamide and oxazol group.

\section{Acknowledgment}

The authors are grateful to the DGRSDT at the MESRS (Algerian Ministry of higher Education and Scientific Research) for financial supports.

\section{Declaration}

\section{Funding:}

The DGRSDT at the MESRS (Algerian Ministry of higher Education and Scientific Research) for financial supports.

\section{Conflicts of interest/Competing interests:}

I did this work on my own, so there was no conflict of interest.

\section{Availability of data and material}

My work is a theoretical study.

\section{Code availability:}

Software application is Gaussian 09, Multiwfn 3.7, and VMD.

\section{Authors' contributions}

Not applicable.

\section{References}

1. Kong, J., et al., Absolute configuration assignment of (+)-fluralaner using vibrational circular dichroism. Chirality, 2017. 29(12): p. 854-864.

2. Zhang, Z., et al., Enantioselective Detection, Bioactivity, and Metabolism of the Novel Chiral Insecticide Fluralaner. Journal of Agricultural and Food Chemistry, 2020.

3. Liu, D., et al., Toxicity and sublethal effects of fluralaner on Spodoptera litura Fabricius (Lepidoptera: Noctuidae). Pesticide biochemistry and physiology, 2018. 152: p. 8-16. 
4. Etienne, T., et al., $A Q M / M M$ study of the absorption spectrum of harmane in water solution and interacting with DNA: the crucial role of dynamic effects. The Journal of Physical Chemistry B, 2013. 117(17): p. 4973-4980.

5. Etienne, T., et al., $Q M / M M$ modeling of Harmane cation fluorescence spectrum in water solution and interacting with DNA. Computational and Theoretical Chemistry, 2014. 1040: $p$. 367-372.

6. Le Bahers, T., et al., Modeling dye-sensitized solar cells: From theory to experiment. The Journal of Physical Chemistry Letters, 2013. 4(6): p. 1044-1050.

7. Pastore, M., et al., Unveiling the nature of post-linear response Z-vector method for timedependent density functional theory. The Journal of Chemical Physics, 2017. 147(2): p. 024108.

8. Dedeoglu, B., et al., Detection of nitroaromatic explosives based on fluorescence quenching of silafluorene-and silole-containing polymers: A time-dependent density functional theory study. The Journal of Physical Chemistry C, 2014. 118(41): p. 23946-23953.

9. Duchanois, T., et al., Heteroleptic Pyridyl-Carbene Iron Complexes with Tuneable Electronic Properties. European Journal of Inorganic Chemistry, 2014. 2014(23): p. 3747-3753.

10. Zojer, E., et al., Excited state localization in organic molecules consisting of conjugated and nonconjugated segments. The journal of chemical physics, 2000. 113(22): p. 10002-10012.

11. Etienne, T., et al., Fluorene-imidazole dyes excited states from first-principles calculationsTopological insights. Theoretical Chemistry Accounts, 2016. 135(4): p. 111.

12. Dreuw, A. and M. Head-Gordon, Single-reference $a b$ initio methods for the calculation of excited states of large molecules. Chemical reviews, 2005. 105(11): p. 4009-4037.

13. Mayer, I., Using singular value decomposition for a compact presentation and improved interpretation of the CIS wave functions. Chemical physics letters, 2007. 437(4-6): p. 284-286.

14. Very, T., et al., Spectral properties of polypyridyl ruthenium complexes intercalated in DNA: theoretical insights into the surrounding effects of [Ru (dppz)(bpy) 2] 2+. Physical Chemistry Chemical Physics, 2012. 14(36): p. 12496-12504.

15. Monari, A., et al., $A Q M / M M$ study on the spinach plastocyanin: redox properties and absorption spectra. Computational and Theoretical Chemistry, 2012. 990: p. 119-125.

16. Chantzis, A., et al., Theoretical evidence of photo-induced charge transfer from DNA to intercalated ruthenium (II) organometallic complexes. Chemical Physics Letters, 2013. 578: p. 133-137.

17. Ronca, E., et al., Charge-displacement analysis for excited states. The Journal of chemical physics, 2014. 140(5): p. 054110.

18. Plasser, F., M. Wormit, and A. Dreuw, New tools for the systematic analysis and visualization of electronic excitations. I. Formalism. The Journal of chemical physics, 2014. 141(2): p. 024106.

19. Jacquemin, D., et al., What is the "best" atomic charge model to describe through-space charge-transfer excitations? Physical Chemistry Chemical Physics, 2012. 14(16): p. 53835388.

20. Guido, C.A., et al., On the metric of charge transfer molecular excitations: a simple chemical descriptor. Journal of chemical theory and computation, 2013. 9(7): p. 3118-3126.

21. Alipour, M. and S. Damiri, Development of a Novel Index for Analysis of Electronically Excited States. ChemPhysChem, 2017. 18(5): p. 480-487.

22. Casida, M.E., Time-dependent density-functional theory for molecules and molecular solids. Journal of Molecular Structure: THEOCHEM, 2009. 914(1-3): p. 3-18.

23. Ohta, K., et al., Theoretical study of the two-photon absorption properties of several asymmetrically substituted stilbenoid molecules. The Journal of chemical physics, 2007. 127(8): p. 084504.

24. Etienne, T., Transition matrices and orbitals from reduced density matrix theory. The Journal of chemical physics, 2015. 142(24): p. 244103. 
25. Wang, X., P. Yan, and X. Mu, Optical properties of S2 and S3 excited states of protonated schiff-base retinal chromophores in TPA, ECD and ROA. Spectrochimica Acta Part A: Molecular and Biomolecular Spectroscopy, 2020. 228: p. 117532.

26. Tian, C., et al., Optical physics on chiral brominated azapirones: Bromophilone $A$ and $B$. Spectrochimica Acta Part A: Molecular and Biomolecular Spectroscopy, 2020. 242: p. 118780.

27. Fan, J. and M. Sun, Optical properties of kalihinol derivatives in TPA, ECD and ROA. Chemical Physics Letters, 2020. 755: p. 137796.

28. Qiao, W., et al., Photoinduced Charge Transfer in Push/Pull Systems of Two-Photon Absorption. ACS omega, 2020. 5(28): p. 17275-17286.

29. Sun, M., J. Chen, and H. Xu, Visualizations of transition dipoles, charge transfer, and electronhole coherence on electronic state transitions between excited states for two-photon absorption. The Journal of chemical physics, 2008. 128(6): p. 064106.

30. Mu, X., et al., Visualizations of electric and magnetic interactions in electronic circular dichroism and raman optical activity. The Journal of Physical Chemistry A, 2019. 123(37): p. 8071-8081.

31. Mu, X., J. Wang, and M. Sun, Visualization of photoinduced charge transfer and electron-hole coherence in two-photon absorption. The Journal of Physical Chemistry C, 2019. 123(23): p. 14132-14143.

32. $\mathrm{Mu}, \mathrm{X}$. , et al., The nature of chirality induced by molecular aggregation and self-assembly. Spectrochimica Acta Part A: Molecular and Biomolecular Spectroscopy, 2019. 212: p. 188198.

33. Warnke, I. and F. Furche, Circular dichroism: electronic. Wiley Interdisciplinary Reviews: Computational Molecular Science, 2012. 2(1): p. 150-166.

34. Frisch, M., et al., gaussian 09, Revision d. 01, Gaussian. Inc., Wallingford CT, 2009. 201.

35. Furche, F. and R. Ahlrichs, Adiabatic time-dependent density functional methods for excited state properties. The Journal of chemical physics, 2002. 117(16): p. 7433-7447.

36. Yanai, T., D.P. Tew, and N.C. Handy, A new hybrid exchange-correlation functional using the Coulomb-attenuating method (CAM-B3LYP). Chemical physics letters, 2004. 393(1-3): p. 5157.

37. Lu, T. and F. Chen, Multiwfn: a multifunctional wavefunction analyzer. Journal of computational chemistry, 2012. 33(5): p. 580-592.

38. Humphrey, W., A. Dalke, and K. Schulten, VMD: visual molecular dynamics. Journal of molecular graphics, 1996. 14(1): p. 33-38. 
Figures

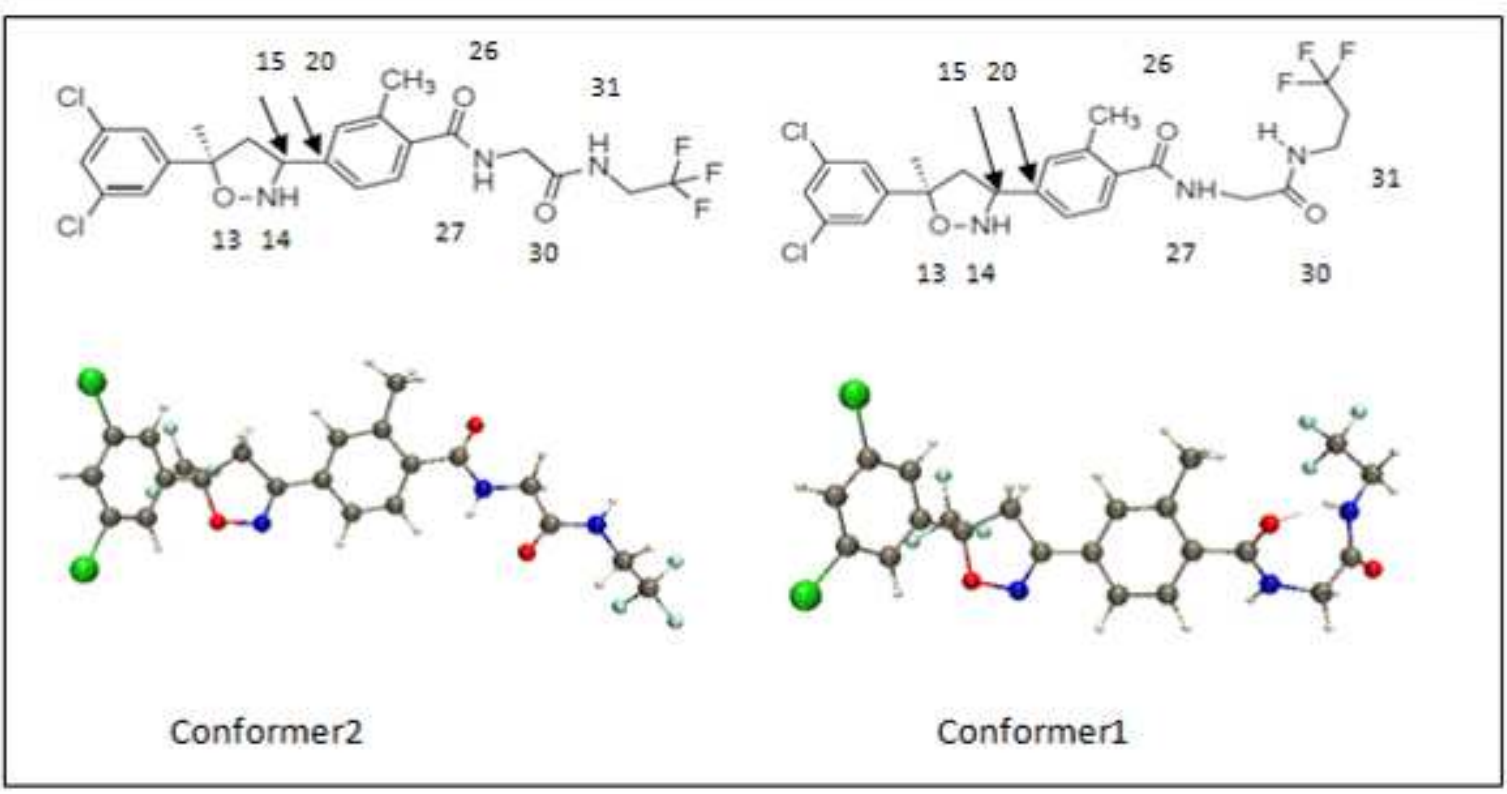

Figure 1

Molecular structure of 4-[(5R)-5-(3, 5-dichlorophenyl)-5-(trifluoromethyl)-4H-1, 2-oxazol-3-yl] -2-methyl-N[2-oxo-2-(2, 2, 2-trifluoroethylamino) ethyl] benzamide.
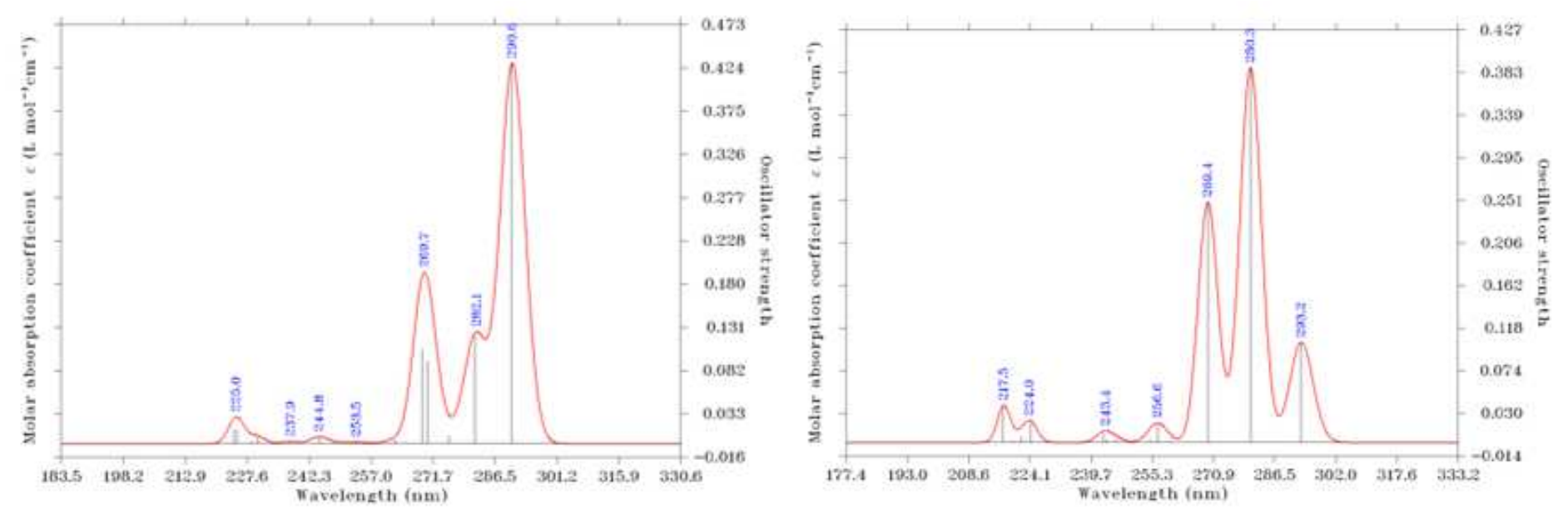

Figure 2

calculated UV-Vis spectra of conformer1 (left) and conformer2 (right) 

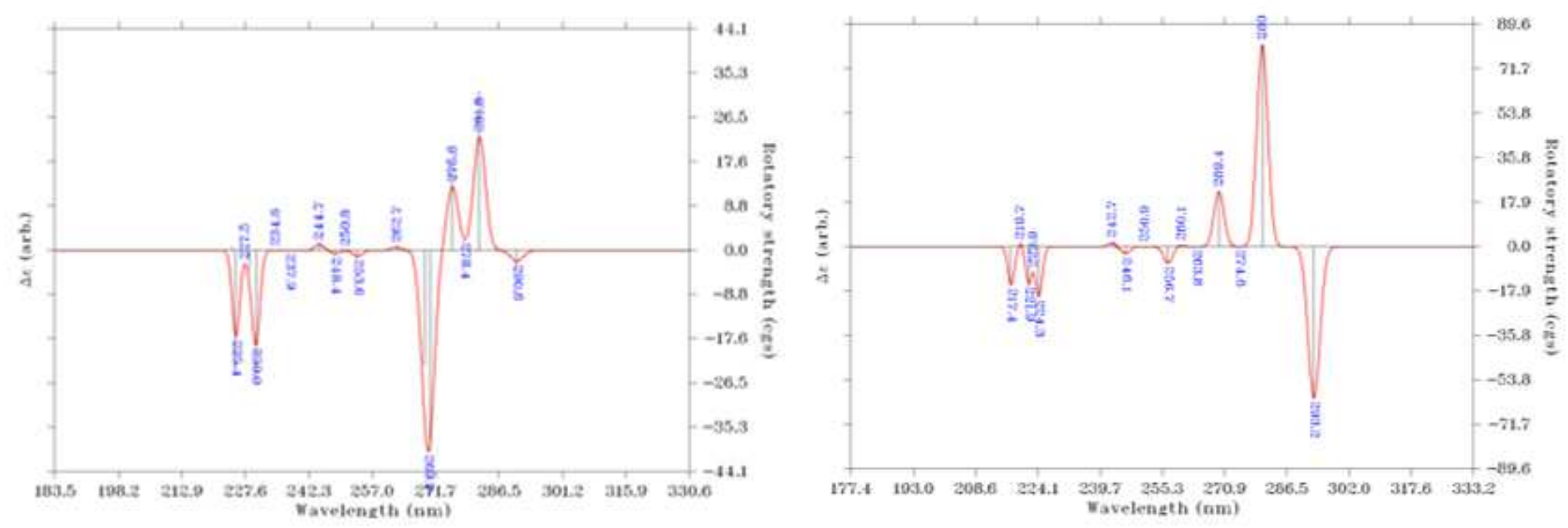

Figure 3

calculated ECD spectra of conformer1 (left) and conformer2 (right)
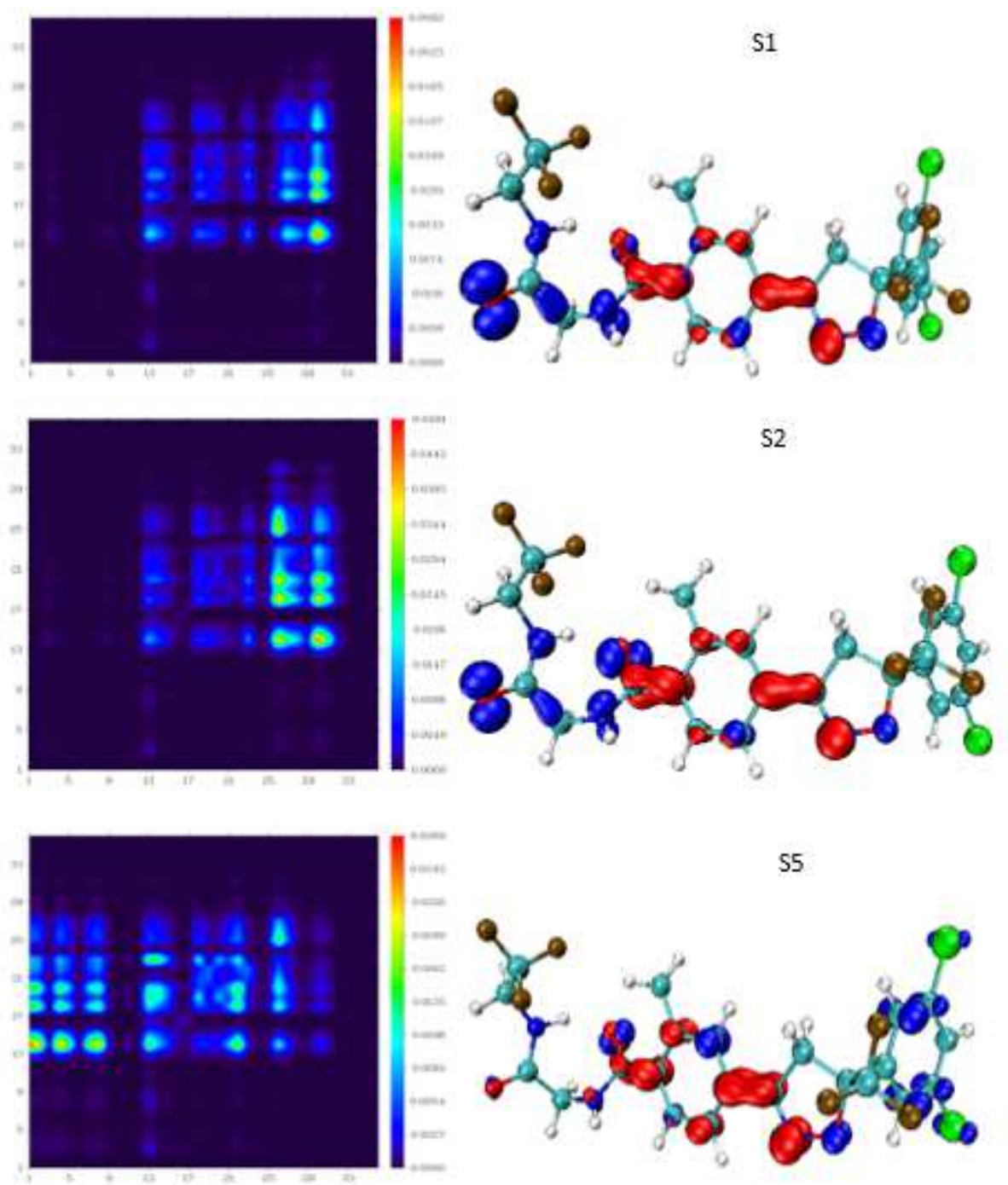

Figure 4 
Transition density matrix (TDM) and charge difference density (CDD) of excited states S1, S2, S5 of conformer1. The isosurface color of the electron density is red while the bleu is hole density.
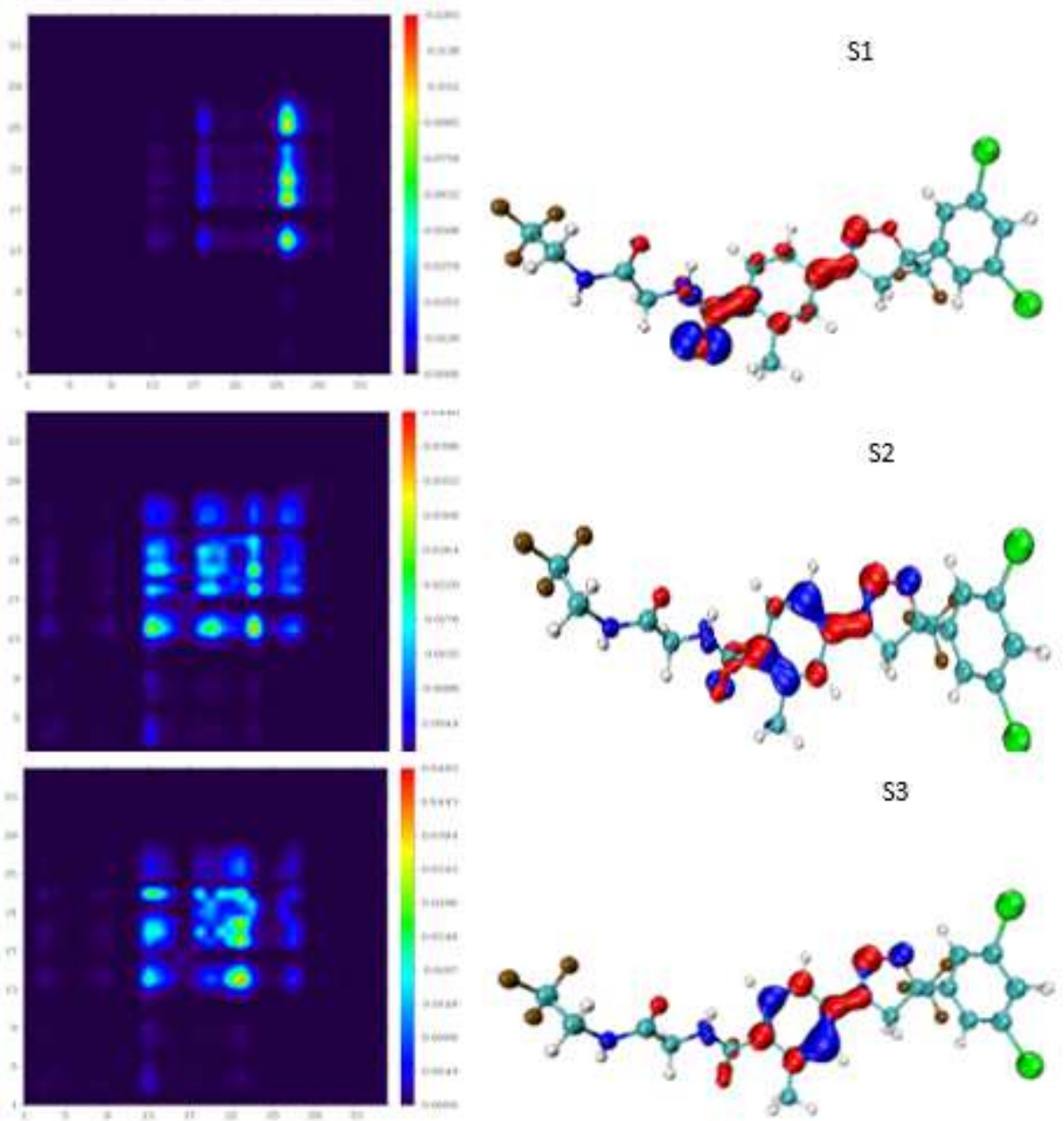

S3

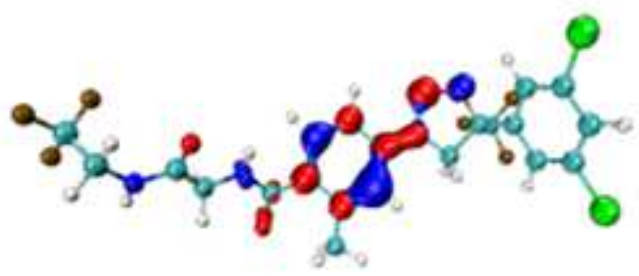

Figure 5

Transition density matrix (TDM) and charge difference density (CDD) of excited states S1, S2, S3 of conformer2. The isosurface color of the electron density is red while the bleu is hole density. 

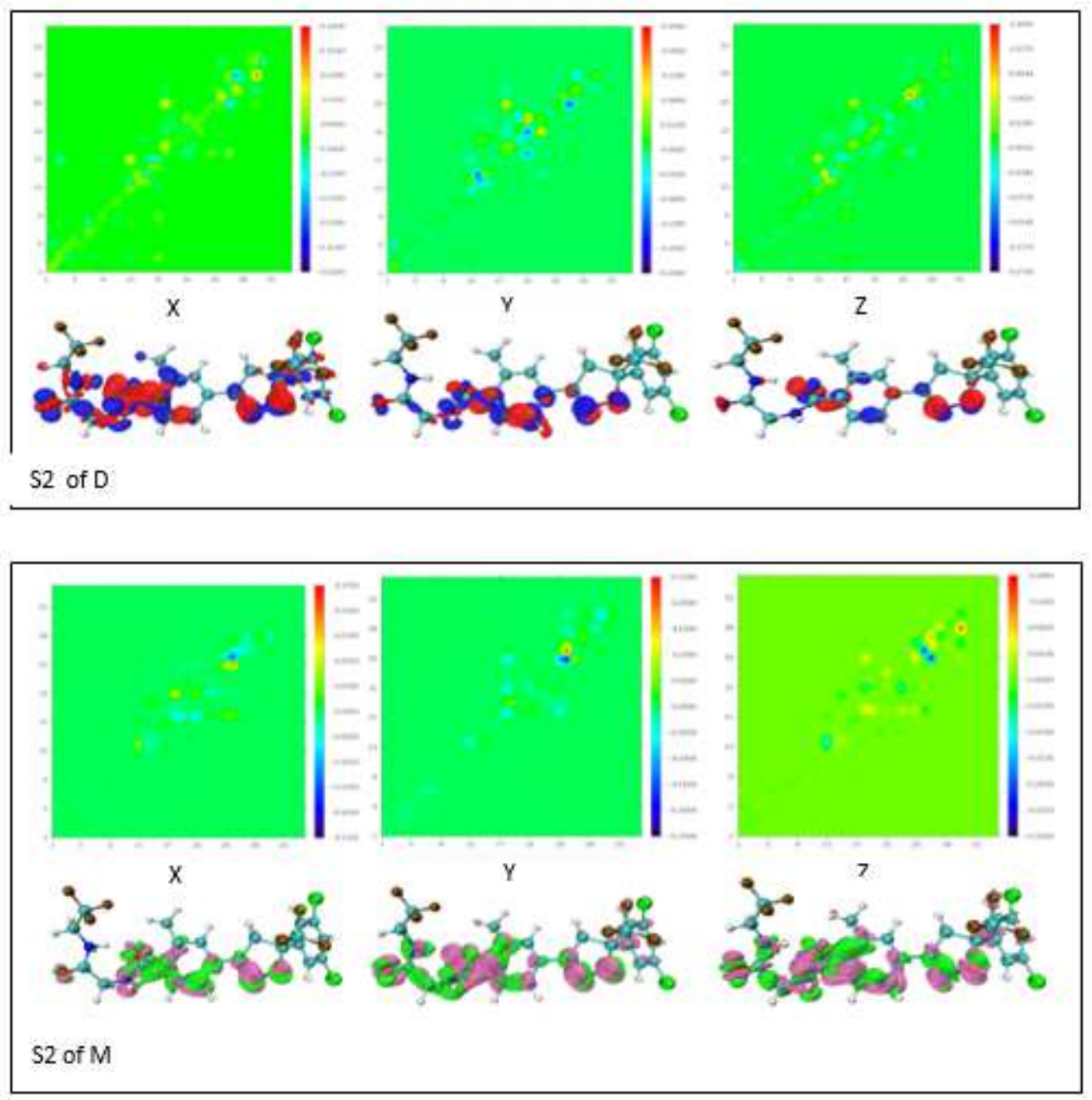

Figure 6

Transition electric dipole moment density (above) and transition magnetic dipole moment density (below) for excited state S2 in ECD of conformer1. 

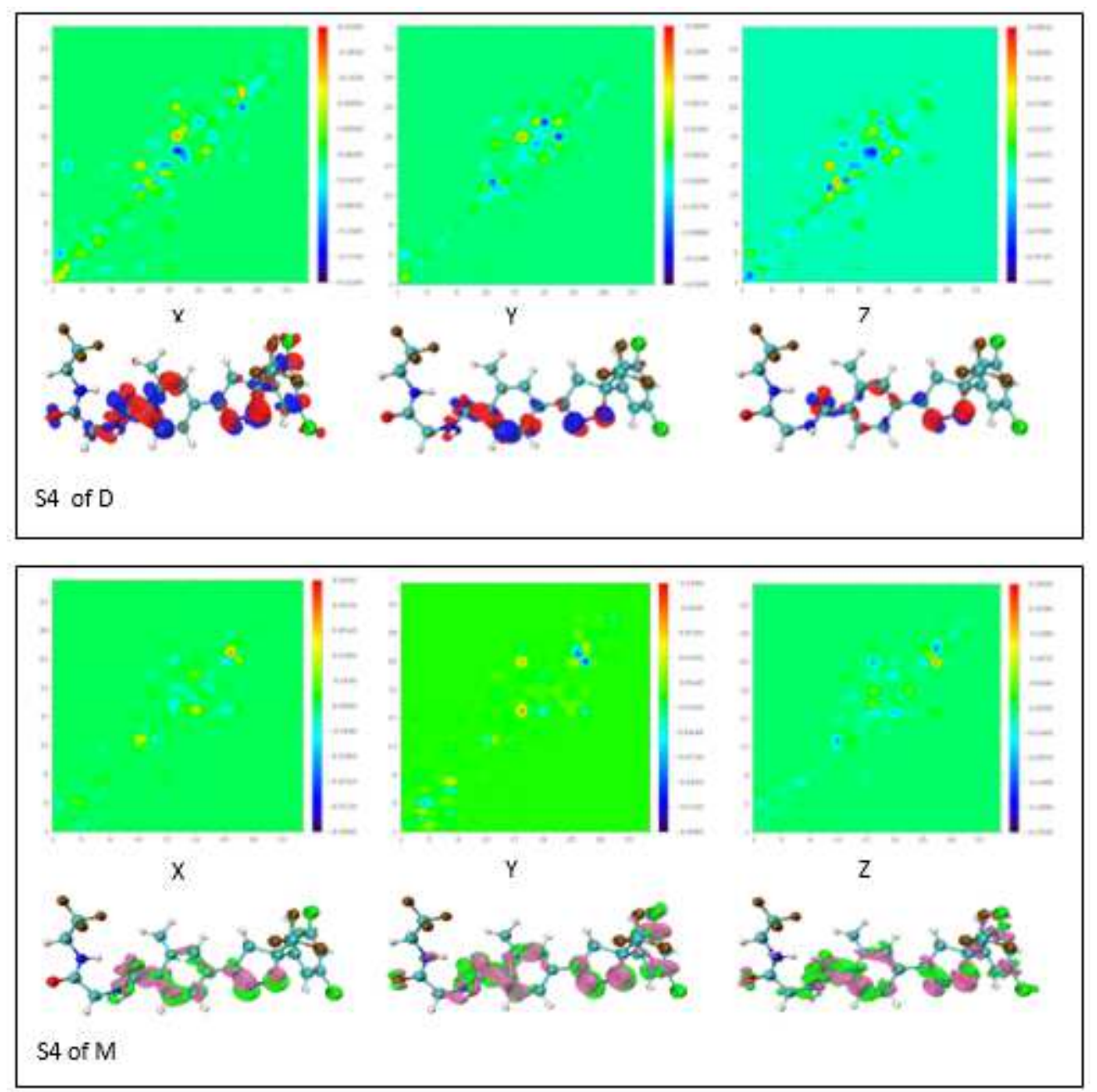

Figure 7

Transition electric dipole moment density (above) and transition magnetic dipole moment density (below) for excited state S4 in ECD of conformer1. 

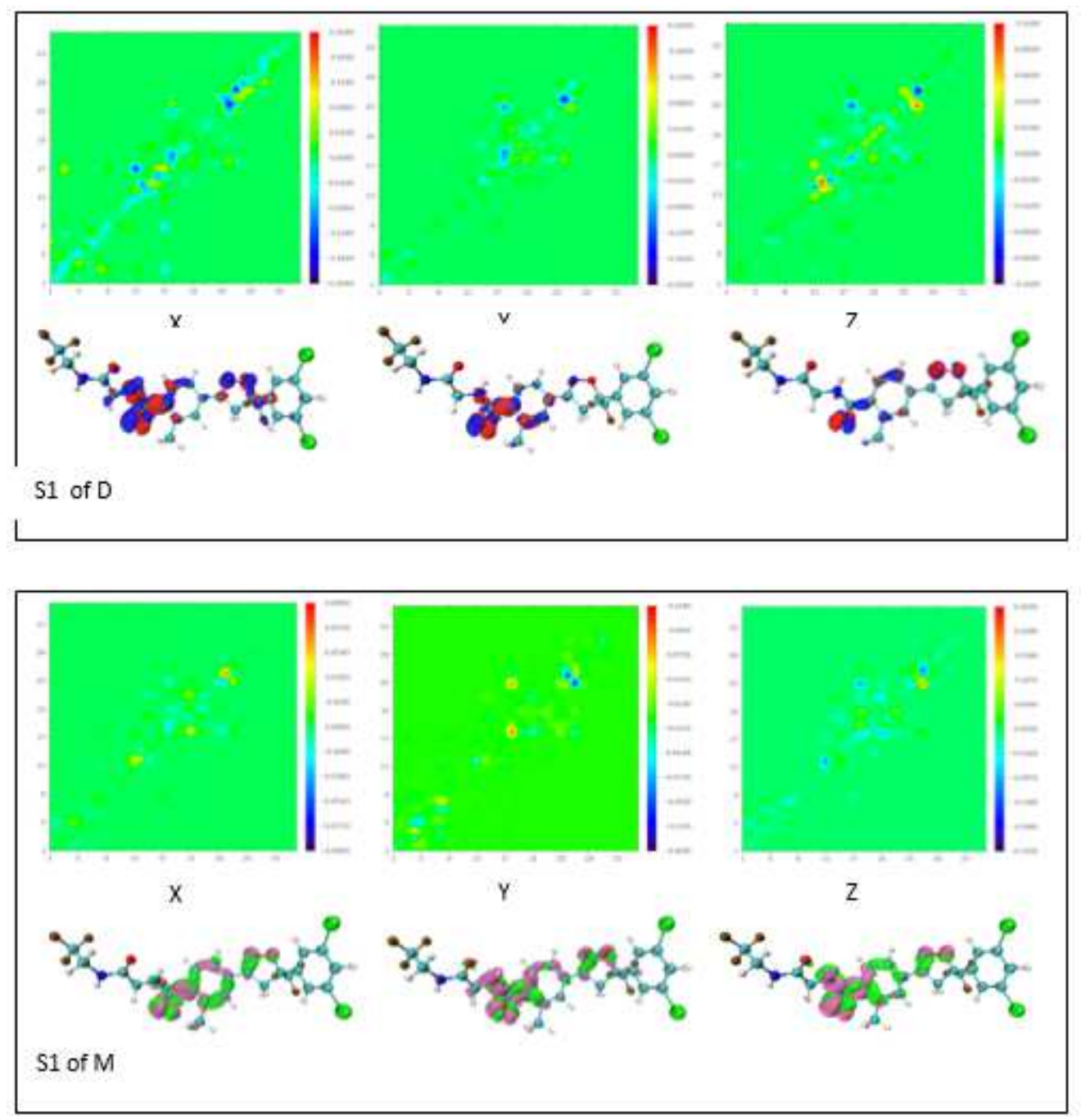

\section{Figure 8}

Transition electric dipole moment density (above) and transition magnetic dipole moment density (below) for excited state S1 in ECD of conformer2. 

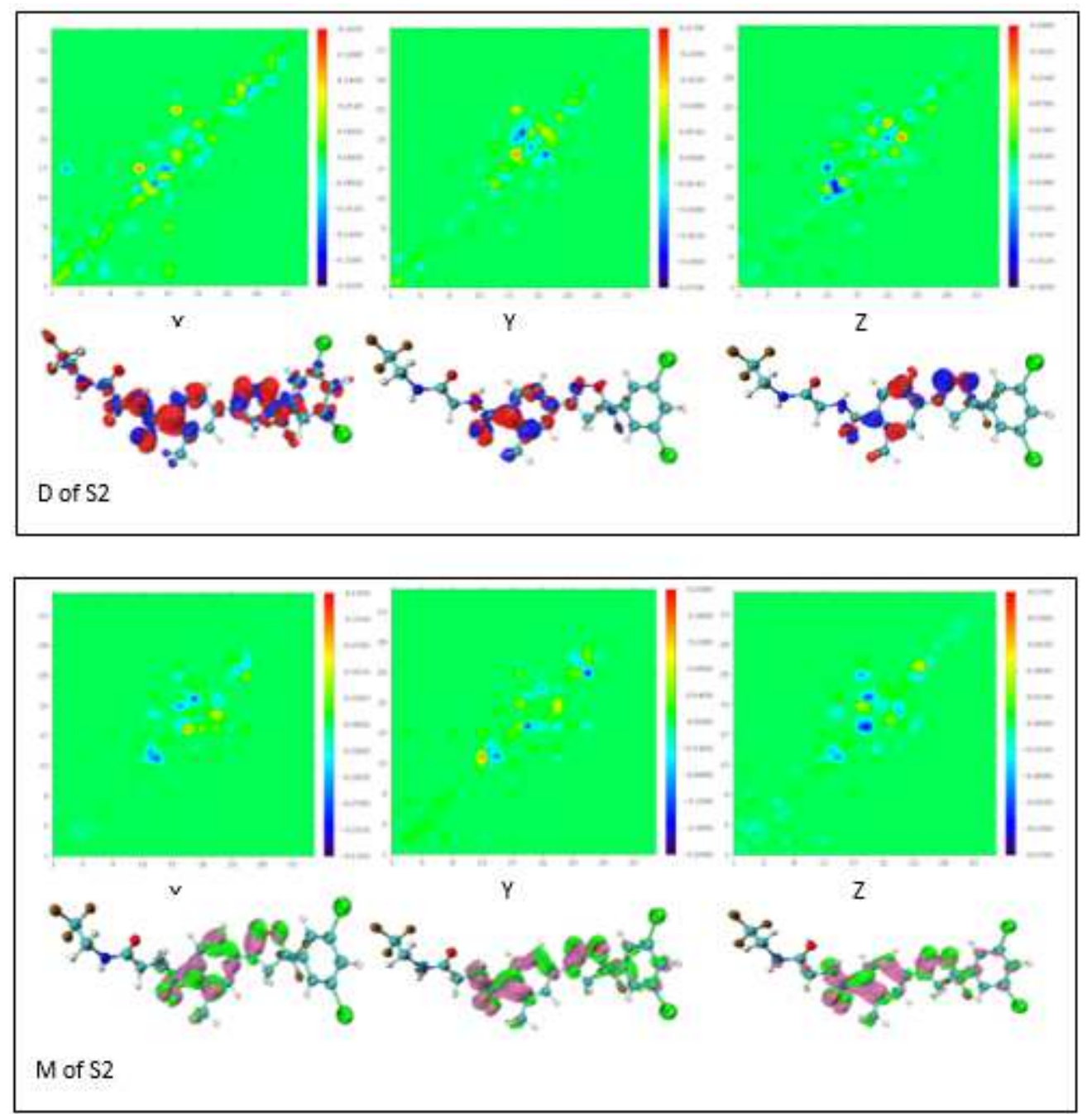

Figure 9

Transition electric dipole moment density (above) and transition magnetic dipole moment density (below) for excited state S2 in ECD of conformer2. 